\title{
Erratum: Longo, U.G., et al. Scapular Dyskinesis: From Basic Science to Ultimate Treatment. International Journal of Environmental Research and Public Health 2020, 17(8), 2974
}

\author{
Umile Giuseppe Longo ${ }^{1, *(1)}$, Laura Risi Ambrogioni ${ }^{1}$, Alessandra Berton ${ }^{1}$, Vincenzo Candela ${ }^{1}$, \\ Carlo Massaroni ${ }^{2}\left(\mathbb{D}\right.$, Arianna Carnevale ${ }^{1,2}{ }^{-0}$, Giovanna Stelitano ${ }^{1}$, Emiliano Schena ${ }^{2}(\mathbb{D}$, \\ Ara Nazarian ${ }^{3}$, Joseph DeAngelis ${ }^{3}(1)$ and Vincenzo Denaro ${ }^{1}$ \\ 1 Department of Orthopaedic and Trauma Surgery, Campus Bio-Medico University, Via Alvaro del Portillo, \\ 200, Trigoria, 00128 Rome, Italy; laura.ambrogioni@gmail.com (L.R.A.); a.berton@unicampus.it (A.B.); \\ v.candela@unicampus.it (V.C.); arianna.carnevale@unicampus.it (A.C.); g.stelitano@unicampus.it (G.S.); \\ denaro@unicampus.it (V.D.) \\ 2 Laboratory of Measurement and Biomedical Instrumentation, Campus Bio-Medico University, Via Alvaro \\ del Portillo, 200, Trigoria, 00128 Rome, Italy; c.massaroni@unicampus.it (C.M.); e.schena@unicampus.it (E.S.) \\ 3 Carl J. Shapiro Department of Orthopaedic Surgery and Center for Advanced Orthopaedic Studies, \\ Beth Israel Deaconess Medical Center, Harvard Medical School, Boston, MA 02215, USA; \\ anazaria@bidmc.harvard.edu (A.N.); jpdeange@bidmc.harvard.edu (J.D.) \\ * Correspondence: g.longo@unicampus.it; Tel.: +39-0622-5411-613; Fax: +39-0622-5411
}

Received: 20 May 2020; Accepted: 25 May 2020; Published: 27 May 2020

The authors would like to correct the names and surnames of the following authors of their previous paper [1], Umile Giuseppe Longo and Laura Risi Ambrogioni.

Therefore, to cite this paper please use the correct reference as follows:

1. Longo, U.G.; Risi Ambrogioni, L.; Berton, A.; Candela, V.; Massaroni, C.; Carnevale, A.; Stelitano, G.; Schena, E.; Nazarian, A.; DeAngelis, J.; et al. Scapular Dyskinesis: From Basic Science to Ultimate Treatment. Int. J. Environ. Res. Public Health 2020, 17, 2974.

\section{Reference}

1. Giuseppe, L.U.; Laura, R.A.; Berton, A.; Candela, V.; Massaroni, C.; Carnevale, A.; Stelitano, G.; Schena, E.; Nazarian, A.; DeAngelis, J.; et al. Scapular Dyskinesis: From Basic Science to Ultimate Treatment. Int. J. Environ. Res. Public Health 2020, 17, 2974. [CrossRef] [PubMed]

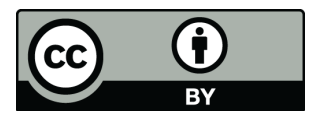

(C) 2020 by the authors. Licensee MDPI, Basel, Switzerland. This article is an open access article distributed under the terms and conditions of the Creative Commons Attribution (CC BY) license (http://creativecommons.org/licenses/by/4.0/). 